\title{
Anti-Cyclic Citrullinated Peptide in Preclinical Rheumatoid Arthritis. Food for Thought
}

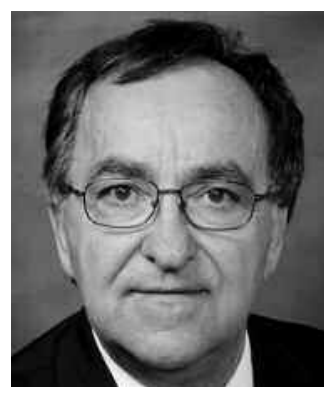

Citrullinated (cit-) epitope detection is an evolving science with different substrates being proposed continuously ${ }^{1-4}$. Given all the published enthusiasm with anti-cyclic citrullinated peptide (CCP), why does the test need to be improved? It is assumed that the cit-epitopes targeted in cit-peptides/proteins are all detected by anti-CCP and are invariable in the various individuals during the phases of disease. That premise may be incorrect.

Recent objective critical evaluation of anti-CCP is severe. Its added value in rheumatoid arthritis (RA) diagnostics, over and above previously existing clinical and laboratory tools, is deemed marginal ${ }^{5}$. The huge anti-CCP literature contains recurring inconsistencies suggesting that authors are using the same test to measure different things in a very heterogeneous disease. Moreover, it is difficult to understand how a test can be associated with more severe evolution in early $\mathrm{RA}^{6}$ and also be positive without arthritis for 10 to 15 years before people get sick ${ }^{7-10}$. How does one reconcile that?

The solution of Chibnik, et al in this issue of The Journal ${ }^{7}$ is to pay more attention to titers of anti-CCP to explain the transition from pre-RA to RA. Just having anti-CCP is not sufficient; also important is how much one has. The higher the titer, the shorter the interval to disease onset! Titers rise steadily until disease onset and then stabilize, as is the current experience in established disease. That the titers rise near RA onset has already been suggested ${ }^{8}$ and is convincingly confirmed here ${ }^{7}$. What does that mean exactly? Either the disease manifests itself only when a sufficient level of autoantibody is reached (quantitative change) or when a given autoantibody emerges whose specificity is associated with disease onset (qualitative change). The 2 explanations are not mutually exclusive, as maturation of an immune response is accompanied by rising titers and epitope dominance.

If the quantitative interpretation is correct, setting a cutoff becomes a double statistical task. Two cutoffs are needed to distinguish normal or non-RA versus RA predisposition versus RA disease. If such fine distinctions are ever possible, the clinician will be more comfortable in reassuring the patients with low titers, as they may never develop
RA or will do so only in 10 to 15 years. On the other hand, he will observe more carefully and maybe treat more aggressively those with rising (high) titers, as these patients are going to get the disease sooner rather than later. Given the prevalence of the HLA shared-epitope alleles and their link to the immune response to citrullinated epitopes ${ }^{11}$, there may be a significant number of people with low to mid-range levels of these autoantibodies. Most will never get sick. How many and who will go on to develop disease cannot be answered by the retrospective study of pre-RA cohorts $^{7-10}$. That question can be answered only prospectively, as in the ongoing study in North American Natives (NAN), which involves RA patients, their healthy first-degree relatives, and healthy unrelated controls ${ }^{12}$. The risk of developing RA in NAN is very high because of the concentration of the permissive gene pool and the harsh environmental challenges. The rate of anti-CCP-positive findings in the 3 groups mentioned is also high at $79 \%$, $20 \%$, and $9 \%$, respectively. Anti-CCP titer differences between the 3 groups were not obviously skewed but will have to be reassessed. Two sets of qualitative differences were observed (see below). Similarly, in early undifferentiated arthritis, anti-CCP titers did not also seem to be predictive of who would develop RA a year later ${ }^{13}$.

If a qualitative autoantibody change occurs near disease onset, that would be easier to resolve with a complementary test to identify the most likely culprit hiding in the proprietary CCP2 mixture ${ }^{14}$. That general need was recognized independently after a recent overview of the anti-CCP literature $^{5}$. Such a test already exists. In the NAN cohort ${ }^{12}$, the rate of anti-Sa (cit-vimentin) was $51 \%, 0 \%$, and $0 \%$ in the groups mentioned. Strictly disease related! Only one anti-CCP-positive healthy person (a relative) developed a RA-like arthritis during the first 3 years of the study. That patient seroconverted to anti-Sa-positive just before disease onset. Nobody else seroconverted or developed disease! Another qualitative aspect was measured in that cohort. RA patients used significantly more anti-CCP immunoglobulin isotypes than the anti-CCP-positive healthy relatives and unrelated controls. The anti-Sa-positive RA patients were

See Threshold cutpoints vs continuous measures of anti-CCP in predicting RA, page 706

Personal non-commercial use only. The Journal of Rheumatology Copyright @ 2009 . All rights reserved. 
largely responsible for the extra anti-CCP isotype usage. Thus, RA patients constituted at least 2 different groups of patients who were anti-CCP-positive: those behaving like healthy people (very little isotype usage) were anti-Sa-negative and those behaving like true RA (more usage) were anti-Sa-positive. No similar data exist for the other cit-protein autoantigens. That extra usage of anti-CCP2 isotypes was also seen in patients with early undifferentiated arthritis, but only in those who developed RA after 1 year ${ }^{13}$. Anti-Sa was not tested in that cohort.

As space does not allow discussion of other legitimate candidates here, I will only summarize why anti-Sa should be the logical complement to anti-CCP ${ }^{15}$. Citrullinated vimentin $^{2,14}$ is generated during apoptosis, like most autoantigens ${ }^{16}$. Cit-vimentin peptides have preferential interaction with shared-epitope HLA alleles ${ }^{11}$. RA pannus is loaded with natural and altered cleavage products of cit-vimentin isoforms ${ }^{2,17}$, some of which may be mutated neoantigens produced during inflammation ${ }^{3}$. The qualitative explanation reflects an ongoing immune response and recoups the quantitative one, as anti-Sa-positive means higher anti-CCP titer ${ }^{15}$.

The overall serological situation can be compared to that of systemic lupus erythematosus (SLE). I submit that anti-CCP is to RA what antinuclear antibody is to SLE. Both are screening tests almost always present but not ipso facto diagnostic. Indeed, both are mostly useful for their negative predictive value. When they are positive and disease-associated, one or more dominant specificities is identified. I posit that anti-Sa is to RA what anti-dsDNA is to SLE. Indeed, anti-Sa has emerged as strictly linked to disease or disease onset ${ }^{2,12}$. It is a better tool in early arthritis to predict persistent and severe disease than anti-CCP and rheumatoid factor put together ${ }^{18,19}$. Finally, anti-Sa titers vary closely and reliably in individual patients with disease activity and adequate response to some treatments ${ }^{19,20}$, like a pathogenic antibody should, and unlike anti-CCP. My prediction is that anti-Sa would best separate patients at high and low risk in the 2 anti-CCP-positive preclinical groups ${ }^{7}$. Unfortunately, none of the sera used in military or American female cohorts could be made available for anti-Sa testing. That would have either challenged or confirmed, and in any case clarified, the conclusions of the authors on how to interpret and deal clinically with people with low versus high titers.

HENRI A. MÉNARD, MD,

Professor of Medicine,

Division of Rheumatology,

McGill University Health Center,

Montreal, Quebec, Canada

Address reprint requests to Dr. H.A. Ménard, Division of Rheumatology, McGill University Health Center, 1650 Cedar Avenue, Montreal, Québec, H3G 1A4, Canada; E-mail: henri.a.menard@muhc.mcgill.ca Supported by Canadian Institutes for Health Research.

\section{REFERENCES}

1. Schellekens GA, Visser H, de Jong BAW, et al. The diagnostic properties of RA antibodies recognizing a cyclic citrullinated peptide. Arthritis Rheum 2000;43:155-63.

2. Despres N, Boire G, Lopez LF, Ménard HA. The Sa system: a novel antigen-antibody system specific for RA. J Rheumatol 1994;21:1027-33

3. Bang H, Egerer K, Gauliard A, et al. Mutation and citrullination modifies vimentin to a novel autoantigen for rheumatoid arthritis. Arthritis Rheum 2007;56:2503-11.

4. Vincent C, Senshu T, Serre G. The major synovial targets of the RA-specific antifilaggrin autoantibodies are deiminated forms of the alpha- and beta-chains of fibrin. J Immunol 2001;166:4177-84

5. Shmerling RS. Testing for anti-CCP antibodies. Is it time to set this genie free? Arch Intern Med 2009;169:9-14

6. Vencovsky J, Machacek S, Sedova L, et al. Autoantibodies can be prognostic markers of an erosive disease in early RA. Ann Rheum Dis 2003;62:427-30.

7. Chibnik LB, Mandl LA, Costenbader KH, Schur PH, Karlson EW Comparison of threshold cut-points and continuous measures of anti-CCP antibodies in predicting future RA. J Rheumatol 2009;36:706-11.

8. Rantapaa-Dahlqvist, de Jong BA, Berglin E, et al. Antibodies against citrullinated peptide and $\operatorname{IgA}$ rheumatoid factor predict the development of rheumatoid arthritis. Arthritis Rheum 2003;48:2741-9.

9. Nielen MMJ, Van Schaardenburg D, Reesink HWR, et al. Specific autoantibodies precede the symptoms of rheumatoid arthritis: a study of serial measurements in blood donors. Arthritis Rheum 2004;50:380-6.

10. Majka DS, Deane KD, Parrish LA, et al. Duration of preclinical RA-related autoantibody positivity increases in subjects with older age at time of disease diagnosis. Ann Rheum Dis 2008;67:801-7.

11. Hill JA, Southwood S, Sette A, Jevnikar AM, Bell DA, Cairns E. Cutting edge: The conversion of arginine to citrulline allows for a high-affinity peptide interaction with the RA-associated HLA-DRB 1*0401 MHC class II molecule. J Immunol 2003; $171: 538-41$

12. Ioan-Facsinay A, Willemze A, Robinson DB, et al. Marked differences in fine specificity and isotype usage of the anti-citrullinated protein antibody in health and disease. Arthritis Rheum 2008;58:3000-8.

13. Verpoort KN, Jol-van der Zijde CM, Papendrecht-van der Voort EA, et al. Isotype distribution of anti-CCP antibodies in undifferentiated arthritis and RA reflects an ongoing immune response. Arthritis Rheum 2006;54:3799-808.

14. Vossenaar ER, Després N, Lapointe E, et al. RA specific anti-Sa antibodies target citrullinated vimentin. Arthritis Res Ther 2004;6:R142-50.

15. Menard HA. Anti-CCP versus anti-Sa antibodies for the diagnosis of RA. Nat Clin Pract Rheumatol 2007;3:76-7.

16. Asaga H, Yamada M, Senshu T. Selective deimination of vimentin in calcium ionophore-induced apoptosis of mouse peritoneal macrophages. Biochem Biophys Res Commun 1998;243:641-6.

17. Tilleman K, Van Steendam K, Cantaert T, De Keyser F, Elewaut D, Deforce D. Synovial detection and autoantibody reactivity of processed citrullinated isoforms of vimentin in inflammatory arthritides. Rheumatology 2008;47:597-604.

18. Boire G, Cossette P, Brum-Fernandes AJ, et al. Anti-Sa antibodies and antibodies against CCP are not equivalent as predictors of severe outcomes in patients with recent-onset polyarthritis. Arthritis Res Ther 2005; 7:R592-603.

19. Innala L, Kokkonen H, Eriksson C, Jidell E, Berglin E, Dahlqvist SR. Antibodies against mutated citrullinated vimentin are a better predictor of disease activity at 24 months in early rheumatoid arthritis than antibodies against citric citrullinated peptides. J Rheumatol 2008;35:1002-8.

20. Menard HA. Effects of RA treatments on citrullinated immune systems in vitro and in vivo. 28th European Workshop on Rheumatology Research, March 1, 2008, Toulouse, France. 